\title{
Quadratic Optimization over a Second-Order Cone with Linear Equality Constraints
}

\author{
Xiao-ling Guo • Zhi-bin Deng • Shu-Cherng Fang • \\ Zhen-bo Wang • Wen-xun Xing
}

Received: 3 November 2013 / Revised: 28 November 2013 / Accepted: 2 December 2013 / Published online: 14 December 2013

(C) Operations Research Society of China, Periodicals Agency of Shanghai University, and Springer-Verlag Berlin Heidelberg 2013

\begin{abstract}
This paper studies the nonhomogeneous quadratic programming problem over a second-order cone with linear equality constraints. When the feasible region is bounded, we show that an optimal solution of the problem can be found in polynomial time. When the feasible region is unbounded, a semidefinite programming (SDP) reformulation is constructed to find the optimal objective value of the original problem in polynomial time. In addition, we provide two sufficient conditions, under which, if the optimal objective value is finite, we show the optimal solution of SDP reformulation can be decomposed into the original space to generate an optimal solution of the original problem in polynomial time. Otherwise, a recession direction can be identified in polynomial time. Numerical examples are included to illustrate the effectiveness of the proposed approach.
\end{abstract}

Keywords Quadratic programming - Linear conic programming · Second-order cone $\cdot$ Cone of nonnegative quadratic functions

Mathematics Subject Classification (2010) 90C20 • 90C26 · 15B48

Fang was supported by the US National Science Foundation (No. DMI-0553310). Guo, Wang and Xing were supported by the National Natural Science Foundation of China (Nos. 11171177 and 11371216). Deng was supported by the Edward P. Fitts Fellowship at North Carolina State University.

X.-1. Guo · Z.-b. Wang · W.-x. Xing

Department of Mathematical Sciences, Tsinghua University, Beijing 100084, China

Z.-b. Deng $(\bowtie) \cdot$ S.-C. Fang

Industrial and Systems Engineering Department, North Carolina State University, Raleigh,

NC 27695, USA

e-mail: zdeng2@ncsu.edu 


\section{Introduction}

Consider the following nonhomogeneous quadratic optimization problem over a second-order cone with linear equality constraints (QP-SOC in short):

$$
\begin{aligned}
V_{\mathrm{QP}-S O C}:=\min & F(x)=x^{\mathrm{T}} Q x+2 c^{\mathrm{T}} x \\
\text { s.t. } \quad & A x=b, \\
& x \in \mathcal{L},
\end{aligned}
$$

where $Q \in \mathbb{R}^{n \times n}$ is a symmetric matrix which is not necessary to be positive semidefinite, $c \in \mathbb{R}^{n}, A \in \mathbb{R}^{m \times n}, b \in \mathbb{R}^{m}$ and $\mathcal{L}=\left\{x \in \mathbb{R}^{n} \mid \sqrt{x^{\mathrm{T}} H x} \leqslant f^{\mathrm{T}} x\right\}$ is a general second-order cone with $H \in \mathbb{R}^{n \times n}$ being a symmetric positive definite matrix and $f \in \mathbb{R}^{n}$ (see [22]).

The second-order cone is a special Bishop-Phelps cone, which has been proven to be useful in functional analysis and vector optimization [4, 9]. A class of cardinalityconstrained portfolio selection problems can also be reformulated as a second-order cone constrained quadratic optimization problem $[7,18]$. Some studies of optimization problems over $\mathcal{L}$ can be found in [1-3, 6]. Recently, linear conic programming has received much attention for solving quadratic optimization problems [5]. Sturm and Zhang introduced cones of nonnegative quadratic functions and reformulated a nonconvex quadratic program as a linear conic program [21]. Guo et al. [12] construct a sequence of linear conic programs to solve quadratic optimization problems over one first-order cone. Tian et al. provided a computable representation of nonconvex homogeneous quadratic programming problems over a union of second-order cones using linear matrix inequalities (LMIs) [22]. Consequently, polynomial-time interior-point algorithms [15] become applicable for this type of problems. For a general nonhomogeneous quadratic programming problem over a second-order cone, Jin et al. [14] provided an exact computable representation based on LMIs. Although the optimal value of problem (QP-SOC) can be obtained in polynomial time [14], there is no known polynomial-time algorithm in the literature to find the optimal solution of problem (QP-SOC) yet. Eichfelder and Povh studied reformulations of nonconvex quadratic programs over convex cones with linear equalities, the optimal solution of the original problem was not obtained either [9]. In particular, when the feasible region is unbounded, the optimal objective value may be infinite, or the optimal solution is unattainable even when the optimal objective value is finite. In this paper, we intend to address these issues for quadratic optimization problems over a second-order cone with linear equality constraints.

By incorporating the concept of recession cones [17], we deal with the problem depending on the boundedness of its feasible region. For the bounded case, we construct a redundant constraint to bound the second-order cone such that the original problem can be transformed into a lower-dimensional problem with one convex quadratic constraint in addition to the linear equality constraints. An equivalent linear conic program is then derived. Since the cone of nonnegative quadratic functions over one convex quadratic constraint has an explicit representation and decomposition [21], a polynomial-time algorithm applies for finding an optimal solution of the original problem. For the unbounded case, following the work of [10] and [14], we present 
an equivalent semidefinite programming (SDP) reformulation to find the optimal objective value of the original problem in polynomial time. Under two sufficient conditions, if the optimal objective value is finite, we can obtain an optimal solution of the original problem in polynomial time by decomposing the solution of the SDP reformulation. Otherwise, we show a way to find in polynomial time a direction along which the objective value goes to minus infinity.

The rest of the paper is arranged as follows. Notations and preliminaries are presented in Sect. 2. In Sect. 3, we study the problem with a bounded feasible region. A polynomial-time algorithm is presented to find an optimal solution in this case. In Sect. 4, the problem with an unbounded feasible region is studied. A polynomial-time algorithm is presented. Under two sufficient conditions, the algorithm either finds an optimal solution, when the optimal objective value is finite, or finds a recession direction along which the objective value goes to minus infinity. Numerical examples are included to illustrate the proposed approaches in Sect. 5. Conclusion follows in Sect. 6.

\section{Notations and Preliminaries}

Some notations are adopted in this paper. Let $\mathbb{R}_{++}$be the set of positive real numbers, $\mathbb{R}_{+}^{n}$ the first orthant of $\mathbb{R}^{n}$ and $\mathbb{N}_{+}$the set of positive integers. For a vector $x \in \mathbb{R}^{n}$, let $x_{i}$ denote the $i$ th component and $x_{i: j}(1 \leqslant i<j \leqslant n)$ a subvector formed by the elements of $x$ from $x_{i}$ to $x_{j}$. For vectors $a, b \in \mathbb{R}^{n}, a \circ b=\left(a_{1} b_{1}, a_{2} b_{2}, \cdots, a_{n} b_{n}\right)^{\mathrm{T}}$ is the Hadamard product of $a$ and $b . \mathcal{S}^{n}$ denotes the set of all $n \times n$ real symmetric matrices and $\mathcal{S}_{+}^{n}$ the set of all $n \times n$ symmetric positive semidefinite matrices. For a matrix $U \in \mathcal{S}^{n}, U \succeq 0$ means $U$ is positive semidefinite while $U \nsucceq 0$ means $U$ is not positive semidefinite, and $U \succ 0$ means $U$ is positive definite. $\operatorname{Diag}(U)$ denotes an $n$-dimensional vector whose $i$ th element is the $i$ th diagonal element of $U$ and $I_{n}$ the $n \times n$-dimensional identity matrix. For matrices $A$ and $B$ of the same size, denote $A \cdot B=\operatorname{trace}\left(A B^{\mathrm{T}}\right)=\sum_{i} \sum_{j} A_{i j} B_{i j}$, where $A_{i j}$ and $B_{i j}$ denote the elements in the $i$ th row and $j$ th column of $A$ and $B$, respectively. For a cone $C, C^{*}$ denotes its dual cone. For a set $W \subseteq \mathbb{R}^{n}, \operatorname{cl}(W)$ stands for the closure of $W$ and $\operatorname{int}(W)$ the interior of $W$.

\subsection{Properties of General Second-Order Cone}

Several new properties of the general second-order cone are derived in this subsection. Notice that a cone is called a proper cone if and only if it is a pointed, closed and convex cone with a non-empty interior [3]. Then we have the following.

Lemma 2.1 If $\mathcal{L}=\left\{x \in \mathbb{R}^{n} \mid \sqrt{x^{\mathrm{T}} H x} \leqslant f^{\mathrm{T}} x\right\}$ is a proper cone, then $f^{\mathrm{T}} H^{-1} f-$ $1>0$.

Proof Since $\mathcal{L}$ can be seen as the intersection of $x^{\mathrm{T}}\left(H-f f^{\mathrm{T}}\right) x \leqslant 0$ and $f^{\mathrm{T}} x \geqslant$ 0 , we have $H-f f^{\mathrm{T}} \nsucceq 0$. Otherwise, $H-f f^{\mathrm{T}}$ can be decomposed as $B^{\mathrm{T}} B$ for some nonzero matrix $B \in \mathcal{S}^{n}$ and $\mathcal{L} \subseteq\left\{x \in \mathbb{R}^{n} \mid B x=0\right\}$, which contradicts the 
properness of $\mathcal{L}$. Using Schur Lemma [3], we have $\left[\begin{array}{ll}1 & f^{\mathrm{T}} \\ f & H\end{array}\right] \nsucceq 0$, or, equivalently, $f^{\mathrm{T}} H^{-1} f-1>0$.

Lemma 2.2 For a general second-order cone $\mathcal{L}$ with $f^{\mathrm{T}} H^{-1} f-1>0$, its dual $\mathcal{L}^{*}=\left\{x \in \mathbb{R}^{n} \mid \sqrt{x^{\mathrm{T}} H^{-1} x} \leqslant \sqrt{\frac{1}{f^{\mathrm{T}} H^{-1} f-1}}\left(H^{-1} f\right)^{\mathrm{T}} x\right\}$.

Proof Set $U=H^{\frac{1}{2}}$ and $y=U x . \mathcal{L}$ can then be rewritten as $\mathcal{L}=\left\{y \in \mathbb{R}^{n} \mid \sqrt{y^{\mathrm{T}} y} \leqslant\right.$ $\left(U^{-1} f\right)^{\mathrm{T}} y$ \}. According to [19], $U^{-1} f$ can be orthogonally transformed into $\beta=$ $\left(0, \cdots, 0,\left\|U^{-1} f\right\|_{2}\right)$ through the Householder transformation $P=I-2 w w^{\mathrm{T}}$, where $w=\frac{U^{-1} f-\beta}{\left\|\beta-U^{-1} f\right\|_{2}}$ and $P=P^{\mathrm{T}}=P^{-1}$. Setting $z=P y, \mathcal{L}$ is equivalent to $\left\{z \in \mathbb{R}^{n} \mid\right.$ $\left.\sqrt{z^{\mathrm{T}} z} \leqslant\left(P U^{-1} f\right)^{\mathrm{T}} z=\beta^{\mathrm{T}} z\right\}$. Because the dual cone of this standard second-order cone is $\left\{z \in \mathbb{R}^{n} \mid \sqrt{z^{\mathrm{T}} z} \leqslant s\left(e^{n}\right)^{\mathrm{T}} z\right\}$, where $s=\sqrt{\frac{\left\|U^{-1} f\right\|_{2}^{2}}{\left\|U^{-1} f\right\|_{2}^{2}-1}}$ and $e^{n}=(0, \cdots, 0,1)^{\mathrm{T}}$, we have

$$
\begin{aligned}
\mathcal{L}^{*} & =\left\{(P U)^{\mathrm{T}} z \mid \sqrt{z^{\mathrm{T}} z} \leqslant s\left(e^{n}\right)^{\mathrm{T}} z\right\} \\
& =\left\{x \in \mathbb{R}^{n} \mid \sqrt{x^{\mathrm{T}} H^{-1} x} \leqslant \sqrt{\frac{1}{f^{\mathrm{T}} H^{-1} f-1}}\left(H^{-1} f\right)^{\mathrm{T}} x\right\} .
\end{aligned}
$$

Corollary 2.1 $\mathcal{L}$ is a proper cone if and only if $f^{\mathrm{T}} H^{-1} f-1>0$.

Proof When $f^{\mathrm{T}} H^{-1} f-1>0, \mathcal{L}^{*}$ is a pointed cone. Since $\mathcal{L}$ is closed, convex and pointed, $\mathcal{L}=\left(\mathcal{L}^{*}\right)^{*}$ is also solid. Therefore, $\mathcal{L}$ is proper. Together with Lemma 2.1, we know $\mathcal{L}$ is a proper cone if and only if $f^{\mathrm{T}} H^{-1} f-1>0$.

Without loss of generality, in this paper, we always assume that $\mathcal{L}$ is a proper cone. Otherwise, we can discuss the problem in a lower-dimensional space.

\subsection{Properties of Recession Cone}

Definition 2.1 [17] Given a convex set $C \subseteq \mathbb{R}^{n}$ and a direction $d \in \mathbb{R}^{n}$, we say that $C$ recedes in the direction of $d$ if and only if $x+\lambda d \in C$ for every $\lambda \geqslant 0$ and $x \in C$. The set of all such vectors $d$, including $d=0$, is called the recession cone of $C$, denoted as $0^{+} C$.

It is easy to prove the following lemmas by the definition of recession cone.

Lemma $2.30^{+}\left\{x \in \mathbb{R}^{n} \mid A x=b\right\}=\left\{d \in \mathbb{R}^{n} \mid A d=0\right\}$ and $0^{+} \mathcal{L}=\left\{d \in \mathbb{R}^{n} \mid\right.$ $d \in \mathcal{L}\}$, where $\mathcal{L}$ is a second-order cone.

The recession cone has the following property:

Lemma 2.4 [17, Corollary 8.3.3] Given closed convex sets $B$ and $C$ in $\mathbb{R}^{n}$ with nonempty intersection, $0^{+}(B \cap C)=0^{+} B \cap 0^{+} C$. 
According to [17], the boundedness of a closed and convex set can be fully decided by its recession cone.

Lemma 2.5 [17] A non-empty, closed and convex set $C \subseteq \mathbb{R}^{n}$ is bounded if and only if $0^{+} C=\{0\}$.

Following [10] and [9], define Feas(QP-SOC) $:=\{x: x$ is feasible to problem (QP-SOC) . We assume that Feas(QP-SOC) is non-empty, which can be easily verified by solving a second-order cone programming problem. By applying the concept of recession cone, the boundedness of Feas(QP-SOC) is discussed in the next subsection.

\subsection{Boundedness of Feas(QP-SOC)}

Since Feas(QP-SOC) is a closed and convex set, from Lemmas 2.3, 2.4 and 2.5, we have

Lemma 2.6 The recession cone $0^{+}$Feas $(\mathrm{QP}-\mathrm{SOC})=\left\{d \in \mathbb{R}^{n} \mid A d=0, d \in \mathcal{L}\right\}$. Moreover, Feas(QP-SOC) is bounded if and only if $0^{+}$Feas $(\mathrm{QP}-\mathrm{SOC})=\{0\}$.

Notice that when $0^{+}$Feas $(\mathrm{QP}-\mathrm{SOC})=\{0\}$, although the feasible region is bounded, the intersection of each linear equality constraint with $\mathcal{L}$ may be unbounded. However, we can construct a redundant linear equality constraint such that its intersection with $\mathcal{L}$ is a bounded set that contains Feas(QP-SOC).

Lemma 2.7 If $0^{+}$Feas $(\mathrm{QP}-\mathrm{SOC})=\{0\}$, then there exists an $\alpha=\sum_{i=1}^{m} k_{i} \alpha^{i} \in$ $\operatorname{int}\left(\mathcal{L}^{*}\right)$, where $\alpha^{i} \in \mathbb{R}^{n}$ are row vectors of $A$ and $k_{i} \in \mathbb{R}, i=1, \cdots, m$. Moreover, the set $\left\{x \in \mathcal{L} \mid \alpha^{\mathrm{T}} x=\sum_{i=1}^{m} k_{i} b_{i}\right\}$ is bounded.

Proof Let $\mathcal{N}(A)=\left\{d \in \mathbb{R}^{n} \mid A d=0\right\}$, then its orthogonal complementary space $\mathcal{N}(A)^{\perp}=\mathcal{R}\left(A^{\mathrm{T}}\right)=\sum_{i=1}^{m} k_{i} \alpha^{i}$, where $\mathcal{R}\left(A^{\mathrm{T}}\right)$ is the range of $A^{\mathrm{T}}$. For any $x \in \mathcal{L}$, it can be uniquely decomposed as $x=x^{1}+x^{2}$, where $x^{1} \in \mathcal{N}(A)$ and $x^{2} \in \mathcal{R}\left(A^{\mathrm{T}}\right)$. Since $0^{+}$Feas(QP-SOC) $=\{0\}$, i.e., $\mathcal{L} \cap \mathcal{N}(A)=\{0\}$, we know $x^{2}=0$ if and only if $x=0$. Denote the projection of $\mathcal{L}$ into $\mathcal{N}(A)$ as $\mathcal{L}^{1}$ and that into $\mathcal{R}\left(A^{\mathrm{T}}\right)$ as $\mathcal{L}^{2}$. Since $\mathcal{L}$ is a proper cone and $\mathcal{L} \cap \mathcal{N}(A)=\{0\}, \mathcal{L}^{2}$ is also a pointed cone. If not, let $y \in \mathcal{L}^{2}$, $-y \in \mathcal{L}^{2}$ and $y \neq 0$, then there exist $\bar{x}^{1}=y^{1}+y \neq 0 \in \mathcal{L}$ and $\bar{x}^{2}=y^{2}+(-y) \neq$ $0 \in \mathcal{L}$, where $y^{1}, y^{2} \in \mathcal{L}^{1}$. Since $\mathcal{L}$ is a proper cone, $\bar{x}^{1}+\bar{x}^{2}=y^{1}+y^{2} \in \mathcal{L} \cap \mathcal{N}(A)$. By $\mathcal{L} \cap \mathcal{N}(A)=\{0\}$, we have $\bar{x}^{1}=-\bar{x}^{2} \in \mathcal{L}$, which contradicts the fact that $\mathcal{L}$ is a pointed cone. In the lower-dimensional subspace $\mathcal{R}\left(A^{\mathrm{T}}\right)$, denote the dual cone of $\mathcal{L}^{2}$ as $\left(\mathcal{L}^{2}\right)^{*}$. According to the duality theory of a cone [3], $\left(\mathcal{L}^{2}\right)^{*}$ has relative interior points with respect to $\mathcal{R}\left(A^{\mathrm{T}}\right)$. For each relative interior point $z \in\left(\mathcal{L}^{2}\right)^{*}$ and any $x=x^{1}+x^{2} \in \mathcal{L}$ with $x^{1} \in \mathcal{N}(A), x^{2} \in \mathcal{R}\left(A^{\mathrm{T}}\right)$ and $x \neq 0$, we have

$$
z \in \mathcal{R}\left(A^{\mathrm{T}}\right) \quad \text { and } \quad\langle z, x\rangle=\left\langle z, x^{1}\right\rangle+\left\langle z, x^{2}\right\rangle=\left\langle z, x^{2}\right\rangle>0 .
$$

Therefore, $z \in \operatorname{int}\left(\mathcal{L}^{*}\right) \cap \mathcal{R}\left(A^{\mathrm{T}}\right)$. Since $\mathcal{R}\left(A^{\mathrm{T}}\right) \cap \operatorname{int}\left(\mathcal{L}^{*}\right) \neq \varnothing$, we know there exists an $\alpha=\sum_{i=1}^{m} k_{i} \alpha^{i} \in \operatorname{int}\left(\mathcal{L}^{*}\right)$. The recession cone $0^{+}\left\{x \in \mathcal{L} \mid \alpha^{\mathrm{T}} x=\sum_{i=1}^{m} k_{i} b_{i}\right\}=$ 
$\left\{d \in \mathcal{L} \mid \alpha^{\mathrm{T}} d=0\right\}=\{0\}$, consequently, the set $\left\{x \in \mathcal{L} \mid \alpha^{\mathrm{T}} x=\sum_{i=1}^{m} k_{i} b_{i}\right\}$ is bounded.

Based on Lemma 2.7, when Feas(QP-SOC) is bounded, a redundant constraint $\alpha^{\mathrm{T}} x=\sum_{i=1}^{m} k_{i}\left(\left(\alpha^{i}\right)^{\mathrm{T}} x\right)=\sum_{i=1}^{m} k_{i} b_{i}$ can be constructed such that the set $\{x \in \mathcal{L} \mid$ $\left.\alpha^{\mathrm{T}} x=\sum_{i=1}^{m} k_{i} b_{i}\right\}$ is bounded. To obtain this redundant constraint, we need to find an interior $\alpha$ in the dual cone $\mathcal{L}^{*}$. This can be achieved by solving the following convex program:

$$
\begin{array}{ll}
V_{\mathrm{CPP}}:=\min \quad-v \\
\text { s.t. } \quad \alpha=\sum_{i=1}^{m} k_{i} \alpha^{i}, \\
& (\alpha, v) \in\left\{y \in \mathbb{R}^{n+1} \mid \sqrt{y^{\mathrm{T}} \tilde{B} y} \leqslant \tilde{f}^{\mathrm{T}} y\right\}, \\
& 0 \leqslant v \leqslant 1, \quad k_{i} \in \mathbb{R}, \quad i=1, \cdots, m .
\end{array}
$$

where $\tilde{B}=\left[\begin{array}{cc}H^{-1} & 0 \\ 0 & 1\end{array}\right]$ and $\tilde{f}=\sqrt{\frac{1}{f^{\mathrm{T}} H^{-1} f-1}}\left[\begin{array}{c}H^{-1} f \\ 0\end{array}\right]$. Notice that problem (CPP) is a second-order cone program with a linear objective, which can be solved in polynomial time [1].

Corollary 2.2 Feas(QP-SOC) is bounded if and only if the optimal value of problem (CPP) is negative, i.e., $V_{\mathrm{CPP}}<0$.

In the following section, we will develop a polynomial-time algorithm to solve problem (QP-SOC) when its feasible region is bounded.

\section{Bounded Case}

According to Lemma 2.7 and Corollary 2.2, when Feas(QP-SOC) is bounded, problem (QP-SOC) can be equivalently written as

$$
\begin{aligned}
V_{\mathrm{QP}-\mathrm{SOC} 1}:=\min & F(x)=x^{\mathrm{T}} Q x+2 c^{\mathrm{T}} x \\
\text { s.t. } \quad & A x=b, \\
& x \in \mathcal{F}=\mathcal{L} \cap\left\{x \in \mathbb{R}^{n} \mid \alpha^{\mathrm{T}} x=\sum_{i=1}^{m} k_{i} b_{i}\right\} .
\end{aligned}
$$

Note that $\alpha \in \mathcal{L}^{*}$. If $\sum_{i=1}^{m} k_{i} b_{i}=0$ and $b=0$, then Feas(QP-SOC) $=\{0\}$. Otherwise, $\mathcal{F}$ is bounded and has a relative interior point. Moreover, since $\mathcal{L}=\left\{x \in \mathbb{R}^{n} \mid\right.$ $\left.x^{\mathrm{T}}\left(H-f f^{\mathrm{T}}\right) x \leqslant 0, f^{\mathrm{T}} x \geqslant 0\right\}, \mathcal{F}=\left\{x \in \mathbb{R}^{n} \mid x^{\mathrm{T}}\left(H-f f^{\mathrm{T}}\right) x \leqslant 0, f^{\mathrm{T}} x \geqslant 0, \alpha^{\mathrm{T}} x=\right.$ $\left.\sum_{i=1}^{m} k_{i} b_{i}\right\}$. We now show that the constraint $f^{\mathrm{T}} x \geqslant 0$ is redundant in $\mathcal{F}$.

Lemma 3.1 If $\mathcal{F} \neq \varnothing$, then the set $\left\{x \in \mathbb{R}^{n} \mid x^{\mathrm{T}}\left(H-f f^{\mathrm{T}}\right) x \leqslant 0, f^{\mathrm{T}} x<0, \alpha^{\mathrm{T}} x=\right.$ $\left.\sum_{i=1}^{m} k_{i} b_{i}\right\}=\varnothing$. Consequently, $f^{\mathrm{T}} x \geqslant 0$ is redundant in $\mathcal{F}$. 
Proof The recession cone $0^{+} \mathcal{F}=\left\{d \in \mathbb{R}^{n} \mid d \in \mathcal{L}, \alpha^{\mathrm{T}} d=0\right\}$. Since $\alpha$ is an interior point of $\mathcal{L}^{*}, 0^{+} \mathcal{F}=\{0\}$. Suppose $x_{0} \in \mathcal{F}$, if there exists a $y_{0} \in \mathbb{R}^{n}$ such that $y_{0}^{\mathrm{T}}\left(H-f f^{\mathrm{T}}\right) y_{0} \leqslant 0, f^{\mathrm{T}} y_{0}<0, \alpha^{\mathrm{T}} y_{0}=\sum_{i=1}^{m} k_{i} b_{i}$, then we have $-y_{0} \in \mathcal{L}$. Since $\mathcal{L}$ is a convex cone, $x_{0}-y_{0} \in \mathcal{L}$ and $\alpha^{\mathrm{T}}\left(x_{0}-y_{0}\right)=\alpha^{\mathrm{T}} x_{0}-\alpha^{\mathrm{T}} y_{0}=0$. Hence, $x_{0}-y_{0} \in$ $0^{+} \mathcal{F}=\{0\}$ and $x_{0}=y_{0}$. This contradicts the fact of $f^{\mathrm{T}} x_{0} \geqslant 0$ and $f^{\mathrm{T}} y_{0}<0$. Therefore, the set $\left\{x \in \mathbb{R}^{n} \mid x^{\mathrm{T}}\left(H-f f^{\mathrm{T}}\right) x \leqslant 0, f^{\mathrm{T}} x<0, \alpha^{\mathrm{T}} x=\sum_{i=1}^{m} k_{i} b_{i}\right\}=\varnothing$ and $f^{\mathrm{T}} x \geqslant 0$ is a redundant constraint in $\mathcal{F}$.

Based on Lemma 3.1, $\mathcal{F}=\left\{x \in \mathbb{R}^{n} \mid x^{\mathrm{T}}\left(H-f f^{\mathrm{T}}\right) x \leqslant 0, \alpha^{\mathrm{T}} x=\sum_{i=1}^{m} k_{i} b_{i}\right\}$ by removing $f^{\mathrm{T}} x \geqslant 0$. Since $\alpha \neq 0$, without loss of generality, we may assume that $\alpha_{1} \neq 0$. Then $x_{1}=\frac{1}{\alpha_{1}}\left(\sum_{i=1}^{m} k_{i} b_{i}-\alpha_{2: n}^{\mathrm{T}} x_{2: n}\right)$. Suppose that $Q=\left[\begin{array}{cc}Q_{11} & q^{\mathrm{T}} \\ q & \bar{Q}\end{array}\right], c=\left[\begin{array}{c}{ }_{1} \\ \bar{c}\end{array}\right]$, $A=\left[\begin{array}{cc}A_{11} & d^{\mathrm{T}} \\ a & \bar{A}\end{array}\right], \alpha=\left[\begin{array}{c}\alpha_{1} \\ \bar{\alpha}\end{array}\right]$ and $H-f f^{\mathrm{T}}=\left[\begin{array}{cc}u & h^{\mathrm{T}} \\ h & \bar{H}\end{array}\right]$, then problem (QP-SOC1) can be reformulated as an equivalent quadratic program in the $(n-1)$-dimensional subspace.

$$
\begin{aligned}
V_{\mathrm{QP}-\mathrm{SOC} 2}:=\min & \tilde{F}(y)=y^{\mathrm{T}} \tilde{Q} y+2 \tilde{c}^{\mathrm{T}} y \\
\text { s.t. } \quad & \tilde{A} y=\tilde{b}, \\
& y^{\mathrm{T}} \tilde{H} y+2 \tilde{d}^{\mathrm{T}} y+\tilde{t} \leqslant 0, \\
& y \in \mathbb{R}^{n-1},
\end{aligned}
$$

where

$$
\begin{aligned}
& \tilde{Q}=\bar{Q}-\frac{1}{\alpha_{1}} \bar{\alpha} q^{\mathrm{T}}-\frac{1}{\alpha_{1}} q \bar{\alpha}^{\mathrm{T}}+\frac{Q_{11}}{\alpha_{1}^{2}} \bar{\alpha} \bar{\alpha}^{\mathrm{T}}, \quad \tilde{c}=\bar{c}-\frac{Q_{11} s}{\alpha_{1}} \bar{\alpha}-\frac{c_{1}}{\alpha_{1}} \bar{\alpha}+s q, \\
& \tilde{A}=\left[\begin{array}{c}
d^{\mathrm{T}}-\frac{A_{11}}{\alpha_{1}} \bar{\alpha}^{\mathrm{T}} \\
\bar{A}-\frac{1}{\alpha_{1}} a \bar{\alpha}^{\mathrm{T}}
\end{array}\right], \quad \tilde{b}=b-\left[\begin{array}{c}
A_{11} s \\
s a
\end{array}\right] \\
& \tilde{H}=\bar{H}-\frac{1}{\alpha_{1}} \bar{\alpha} h^{\mathrm{T}}-\frac{1}{\alpha_{1}} h \bar{\alpha}^{\mathrm{T}}+\frac{u}{\alpha_{1}^{2}} \bar{\alpha} \bar{\alpha}^{\mathrm{T}}, \\
& \tilde{d}=-\frac{u s}{\alpha_{1}} \bar{\alpha}+s h, \quad \tilde{t}=u s^{2}, \quad s=\frac{\sum_{i=1}^{m} k_{i} b_{i}}{\alpha_{1}} .
\end{aligned}
$$

Notice that there is a constant difference of $Q_{11} s^{2}+2 c_{1} s$ between the optimal objective values of $V_{\mathrm{QP}-\mathrm{SOC} 1}$ and $V_{\mathrm{QP}-\mathrm{SOC} 2}$.

Denote $\tilde{\mathcal{F}}=\left\{y \in \mathbb{R}^{n-1} \mid y^{\mathrm{T}} \tilde{H} y+2 \tilde{d}^{\mathrm{T}} y+\tilde{t} \leqslant 0\right\}$, then we have the next lemma.

Lemma 3.2 If Feas(QP-SOC) is bounded with a non-empty relative interior, then $\tilde{F}$ is bounded and has an interior point. Moreover, $\tilde{H} \succeq 0$ in problem (QP-SOC2).

Proof Since the feasible region $\mathcal{F}$ in problem (QP-SOC1) is non-empty and bounded with relative interior points, $\tilde{F}$, the projection of $\mathcal{F}$ into an $(n-1)$-dimensional subspace, is bounded and has an interior point [11]. Suppose that $\tilde{H}$ has a negative eigenvalue $\lambda_{0}$ with $y_{0}$ being the corresponding eigenvector, then

$$
\left(\beta y_{0}\right)^{\mathrm{T}} \tilde{H}\left(\beta y_{0}\right)+2 \tilde{d}^{\mathrm{T}}\left(\beta y_{0}\right)+\tilde{t}=\lambda_{0} \beta^{2}\left\|y_{0}\right\|_{2}^{2}+2 \beta \tilde{d}^{\mathrm{T}} y_{0}+\tilde{t},
$$


which goes to minus infinity as $\beta \rightarrow \infty$. This causes a contradiction to the boundedness of $\mathcal{F}$. Hence we have $\tilde{H} \succeq 0$.

Therefore, for problem (QP-SOC) with a bounded feasible region, we can obtain its optimal solution by solving problem (QP-SOC2). Here we first reformulate the problem into a linear conic program and then decompose the optimal solution in the matrix space to get an optimal solution of problem (QP-SOC2).

Definition 3.1 [21] Given a set $\mathcal{W} \subseteq \mathbb{R}^{n}$, the cone of nonnegative quadratic functions over $\mathcal{W}$ is defined as

$$
D_{\mathcal{W}}=\left\{\left[\begin{array}{cc}
z_{0} & z^{\mathrm{T}} \\
z & Z
\end{array}\right] \in \mathcal{S}^{n+1} \mid x^{\mathrm{T}} Z x+2 z^{\mathrm{T}} x+z_{0} \geqslant 0, \forall x \in \mathcal{W}\right\} .
$$

Its dual cone $D_{\mathcal{W}}^{*}$ is

$$
D_{\mathcal{W}}^{*}=\operatorname{cl}\left(\left\{\sum_{i} \lambda_{i}\left[\begin{array}{c}
1 \\
x^{i}
\end{array}\right]\left[\begin{array}{c}
1 \\
x^{i}
\end{array}\right]^{\mathrm{T}}: \lambda_{i} \geqslant 0, x^{i} \in W\right\}\right),
$$

which is the closed convex cone generated by

$$
\mathcal{G}=\left\{X=\left[\begin{array}{l}
1 \\
x
\end{array}\right]\left[\begin{array}{l}
1 \\
x
\end{array}\right]^{\mathrm{T}} \in \mathcal{S}^{n+1}: x \in W\right\} .
$$

Eichfelder and Povh proved that, for a closed and bounded set $W$, under some conditions, the quadratic optimization problem over $W$ together with several linear equalities is equivalent to a linear conic program over the cone $D_{W}^{*}$ in the sense that they have the same optimal value. The linear constraints can be transformed into LMIs in the conic program [10]. A more detailed discussion can be found in [14]. As a direct corollary of Lemma 8 in [14], we have the next theorem.

Theorem 3.1 The optimal value $V_{Q P-S O C 2}$ is equal to the optimal value of

$$
\begin{aligned}
& V_{\mathrm{LCoP} 1}:=\min {\left[\begin{array}{cc}
0 & \tilde{c}^{\mathrm{T}} \\
\tilde{c} & \tilde{Q}
\end{array}\right] \cdot\left[\begin{array}{cc}
1 & y^{\mathrm{T}} \\
y & Y
\end{array}\right] } \\
& \text { s.t. } \quad \tilde{A} y=\tilde{b}, \quad \operatorname{Diag}\left(\tilde{A} Y \tilde{A}^{\mathrm{T}}\right)=\tilde{b} \circ \tilde{b}, \\
& {\left[\begin{array}{cc}
1 & y^{\mathrm{T}} \\
y & Y
\end{array}\right] \in D_{\tilde{\mathcal{F}}}^{*} . }
\end{aligned}
$$

Notice that $\tilde{\mathcal{F}}$ is a convex quadratic constraint. According to [21], $D_{\tilde{\mathcal{F}}}^{*}$ has explicit LMI representations and decompositions.

Lemma $3.3\left[21\right.$, Theorem 1] For $\tilde{\mathcal{F}}=\left\{y \in \mathbb{R}^{n-1} \mid y^{\mathrm{T}} \tilde{H} y+2 \tilde{d}^{\mathrm{T}} y+\tilde{t} \leqslant 0\right\}$,

$$
D_{\tilde{\mathcal{F}}}^{*}=\left\{Z \in \mathcal{S}_{+}^{n} \mid\left[\begin{array}{cc}
\tilde{t} & \tilde{d}^{\mathrm{T}} \\
\tilde{d} & \tilde{H}
\end{array}\right] \cdot Z \leqslant 0\right\} .
$$


Lemma 3.3 shows that problem (LCoP1) is a linear conic program over the cone $\mathcal{S}_{+}^{n}$ with some LMIs, which can be solved in polynomial time. To decompose the optimal solution of problem (LCoP1) to the original Euclidean space for finding the optimal solutions of problem (QP-SOC2), we need the next result.

Lemma 3.4 [21, Proposition 3] Let $B \in \mathcal{S}^{n}$ and $X \in \mathcal{S}_{+}^{n}$. Suppose that $\operatorname{rank}(X)=r$, then $B \cdot X \leqslant 0$ if and only if there exists a rank-one decomposition of $X$ such that $X=\sum_{i=1}^{r} x^{i}\left(x^{i}\right)^{\mathrm{T}}$ and $\left(x^{i}\right)^{\mathrm{T}} B x^{i} \leqslant 0$ for $i=1, \cdots, r$.

Since $\tilde{\mathcal{F}}$ is closed, bounded and convex, by applying the polynomial-time Procedure 1 of [21], the optimal solution $\left[\begin{array}{ll}1 & y^{\mathrm{T}} \\ y & Y\end{array}\right] \in D_{\tilde{\mathcal{F}}}^{*}$ can be decomposed as

$$
\left[\begin{array}{cc}
1 & y^{\mathrm{T}} \\
y & Y
\end{array}\right]=\sum_{i=1}^{p} \lambda_{i}\left[\begin{array}{c}
1 \\
y^{i}
\end{array}\right]\left[\begin{array}{c}
1 \\
y^{i}
\end{array}\right]^{\mathrm{T}}
$$

where $y^{i} \in \tilde{\mathcal{F}}, \lambda_{i} \geqslant 0, i=1, \cdots, p$. Moreover, we can derive from the arguments of [10] that if we can decompose the solution of the linear conic program in the matrix space to the original Euclidean space, the linear constraints are satisfied automatically. The result is stated as follows:

Theorem 3.2 For the optimal solution $\left[\begin{array}{ll}1 & y^{\mathrm{T}} \\ y & Y\end{array}\right]$ of problem $(\mathrm{LCoP} 1)$, if $\left[\begin{array}{ll}1 & y^{\mathrm{T}} \\ y & Y\end{array}\right]=$ $\sum_{i=1}^{p} \lambda_{i}\left[\begin{array}{c}1 \\ y^{i}\end{array}\right]\left[\begin{array}{l}1 \\ y^{i}\end{array}\right]^{\mathrm{T}}$ with $\lambda_{i} \geqslant 0, y^{i} \in \tilde{\mathcal{F}}$, then $\tilde{A} y^{i}=\tilde{b}, i=1, \cdots, p$, and each $y^{i}$ is an optimal solution of problem (QP-SOC2).

Proof If $\left[\begin{array}{cc}1 & y^{\mathrm{T}} \\ y & Y\end{array}\right]=\sum_{i=1}^{p} \lambda_{i}\left[\begin{array}{c}1 \\ y^{i}\end{array}\right]\left[\begin{array}{l}1 \\ y^{i}\end{array}\right]^{\mathrm{T}}$ is the optimal solution of problem (LCoP1), the constraints of problem (LCoP1) imply that $\sum_{i=1}^{p} \lambda_{i}=1, y=\sum_{i=1}^{p} \lambda_{i} y^{i}$ and $Y=$ $\sum_{i=1}^{p} \lambda_{i} y^{i}\left(y^{i}\right)^{\mathrm{T}}$. Then, for every row vector $\tilde{\alpha}^{j}$ of matrix $\tilde{A}$, we have

$$
\sum_{i=1}^{p} \lambda_{i}\left(\tilde{\alpha}^{j}\right)^{\mathrm{T}} y^{i}=\tilde{b}_{j} \quad \text { and } \quad \sum_{i=1}^{p} \lambda_{i}\left(\left(\tilde{\alpha}^{j}\right)^{\mathrm{T}} y^{i}\right)^{2}=\tilde{b}_{j}^{2} \quad \text { for } j=1, \cdots, m .
$$

It follows that

$$
\begin{aligned}
0 & =\sum_{i=1}^{p} \lambda_{i}\left(\left(\tilde{\alpha}^{j}\right)^{\mathrm{T}} y^{i}\right)^{2}-\left(\sum_{i=1}^{p} \lambda_{i}\left(\tilde{\alpha}^{j}\right)^{\mathrm{T}} y^{i}\right)^{2} \\
& =\sum_{i=1}^{p} \lambda_{i}\left(\left(\tilde{\alpha}^{j}\right)^{\mathrm{T}} y^{i}-\sum_{k=1}^{p} \lambda_{k}\left(\tilde{\alpha}^{j}\right)^{\mathrm{T}} y^{k}\right)^{2} \geqslant 0 .
\end{aligned}
$$

Hence we have

$$
\left(\tilde{\alpha}^{j}\right)^{\mathrm{T}} y^{i}=\sum_{k=1}^{p} \lambda_{k}\left(\tilde{\alpha}^{j}\right)^{\mathrm{T}} y^{k}=\tilde{b}_{j} \quad \text { for } i=1, \cdots, p \text { and } j=1, \cdots, m .
$$


Consequently, $\tilde{A} y^{i}=\tilde{b}$ for all $i=1, \cdots, p$.

Since $y^{i}$ is feasible to problem (QP-SOC2), we have

$$
\begin{aligned}
V_{\mathrm{QP}-\mathrm{SOC} 2} & \leqslant \sum_{i=1}^{p} \lambda_{i}\left(\left(y^{i}\right)^{\mathrm{T}} \tilde{Q} y^{i}+2 \tilde{c}^{\mathrm{T}} y^{i}\right) \\
& =\sum_{i=1}^{p} \lambda_{i}\left[\begin{array}{cc}
0 & \tilde{c}^{\mathrm{T}} \\
\tilde{c} & \tilde{Q}
\end{array}\right] \cdot\left[\begin{array}{cc}
1 & \left(y^{i}\right)^{\mathrm{T}} \\
y^{i} & y^{i}\left(y^{i}\right)^{\mathrm{T}}
\end{array}\right] \\
& =\left[\begin{array}{cc}
0 & \tilde{c}^{\mathrm{T}} \\
\tilde{c} & \tilde{Q}
\end{array}\right] \cdot\left(\sum_{i=1}^{p} \lambda_{i}\left[\begin{array}{c}
1 \\
y^{i}
\end{array}\right]\left[\begin{array}{c}
1 \\
y^{i}
\end{array}\right]^{\mathrm{T}}\right)=V_{\mathrm{LCoP} 1}=V_{\mathrm{QP}-\mathrm{SOC} 2},
\end{aligned}
$$

where the last equality follows from Theorem 3.1. Therefore, $V_{\mathrm{QP}-\mathrm{SOC} 2}=\left(y^{i}\right)^{\mathrm{T}} \tilde{Q} y^{i}$ $+2 \tilde{c}^{\mathrm{T}} y^{i}$ for any $i=1, \cdots, p$. Hence, $y^{i}$ is an optimal solution of problem (QP-SOC2) for $i=1, \cdots, p$.

Based on the results obtained in this section, we can design a polynomial-time algorithm to solve problem (QP-SOC) with a bounded feasible region.

\section{Algorithm 1: Polynomial-time algorithm for solving problem (QP-SOC) with a bounded feasible region}

Step 1 For a given problem $(\mathrm{QP}-\mathrm{SOC})$, if Feas $(\mathrm{QP}-\mathrm{SOC})=\varnothing$, return that problem (QP-SOC) is not feasible. Otherwise, calculate the dual cone of $\mathcal{L}$, construct and solve the corresponding second-order programming problem (CPP). If the optimal value of problem (CPP) is 0 or the problem is not feasible, return that Feas(QP$\mathrm{SOC}$ ) is unbounded. Otherwise, record the optimal solution $\alpha, k_{i}, i=1, \cdots, m$, and go to Step 2.

Step 2 Add the redundant constraint $\alpha^{\mathrm{T}} x=\sum_{i=1}^{m} k_{i} b_{i}$ to problem (QP-SOC) and construct problem (QP-SOC2).

Step 3 Construct and solve the equivalent linear conic program (LCoP1). Decompose the optimal solution $\left[\begin{array}{ll}1 & y^{\mathrm{T}} \\ y & Y\end{array}\right]$ of problem (LCoP1) using Procedure 1 in [21] to get $\left[\begin{array}{cc}1 & y^{\mathrm{T}} \\ y & Y\end{array}\right]=\sum_{i=1}^{p} \lambda_{i}\left[\begin{array}{c}1 \\ y^{i}\end{array}\right] \cdot\left[\begin{array}{c}1 \\ y^{i}\end{array}\right]^{\mathrm{T}}$.

Step 4 For each $y^{i}, i=1, \cdots, p$, set $x_{1}^{i}=\frac{1}{\alpha_{1}}\left(\sum_{j=1}^{m} k_{j} b_{j}-\alpha_{2: n}^{\mathrm{T}} y^{i}\right), x_{2: n}^{i}=y^{i}$. Return $x^{i}, i=1, \cdots, p$, as the optimal solutions of problem (QP-SOC).

Since each step in the proposed algorithm can be completed in polynomial time, we can solve problem (QP-SOC) in polynomial time when the feasible region is bounded. Because each $y^{i}$ is an optimal solution to problem (QP-SOC2) according to Theorem 3.2, the corresponding $x^{i}$ in Step 4, $i=1, \cdots, p$, is an optimal solution of problem (QP-SOC).

\section{Unbounded Case}

In this section, we study problem (QP-SOC) when its feasible region is unbounded. We will treat its optimal objective value and optimal solution separately. In Sect. 4.1, 
problem (QP-SOC) is reformulated into an SDP problem. Then the optimal objective value of problem (QP-SOC) can be obtained in polynomial time by solving the equivalent SDP reformulation. In Sect. 4.2, two sufficient conditions are derived for decomposing the optimal solution of SDP reformulation into the original space. Under these two conditions, in Sect. 4.3, an optimal solution of problem (QP-SOC) can be found in polynomial time if the optimal objective value is finite. Otherwise, as shown in Sect. 4.4, a recession direction can be found in polynomial time along which the objective value goes to minus infinity. A polynomial-time algorithm is then proposed to solve problem (QP-SOC) with an unbounded feasible region in Sect. 4.5.

\subsection{Optimal Objective Value}

A polynomial-time algorithm is shown here to obtain the optimal objective value of problem (QP-SOC) using the SDP techniques. Similar to the discussion of Theorem 3.1, problem (QP-SOC) is equivalent to the following linear conic program:

$$
\begin{aligned}
V_{\mathrm{LCoP} 2}:= & \min \left[\begin{array}{ll}
0 & c^{\mathrm{T}} \\
c & Q
\end{array}\right] \cdot\left[\begin{array}{cc}
1 & x^{\mathrm{T}} \\
x & X
\end{array}\right] \\
\text { s.t. } & A x=b, \quad \operatorname{Diag}\left(A X A^{\mathrm{T}}\right)=b \circ b, \\
& {\left[\begin{array}{cc}
1 & x^{\mathrm{T}} \\
x & X
\end{array}\right] \in D_{\mathcal{L}}^{*} . }
\end{aligned}
$$

Our goal is to solve problem (LCoP2) and decompose the optimal solution properly. Recently, Jin et al. [14] provided an LMI representation of the cone of nonnegative quadratic functions over a non-empty set $\left\{(x, y) \in \mathbb{R}^{n_{1}} \times \mathbb{R}^{n_{2}} \mid\|x\|_{2} \leqslant\right.$ $\left.a_{1}+a_{2}^{\mathrm{T}} x+a_{3}^{\mathrm{T}} y\right\}$ with $a_{1} \in \mathbb{R}, a_{2} \in \mathbb{R}^{n_{1}}$ and $a_{3} \in \mathbb{R}^{n_{2}}$. Similarly, we can derive an LMI representation of $D_{\mathcal{L}}^{*}$ as follows:

$$
D_{\mathcal{L}}^{*}=\left\{U=\left[\begin{array}{cc}
\chi & x^{\mathrm{T}} \\
x & X
\end{array}\right] \in \mathcal{S}^{n+1} \mid U \succeq 0, \sqrt{x^{\mathrm{T}} H x} \leqslant f^{\mathrm{T}} x,\left(f f^{\mathrm{T}}-H\right) \cdot X \geqslant 0\right\} .
$$

Problem (QP-SOC) can then be represented as the following equivalent semidefinite program:

$$
\begin{aligned}
V_{\mathrm{SP}}:=\min & \bar{F}(Z)=\left[\begin{array}{ll}
0 & c^{\mathrm{T}} \\
c & Q
\end{array}\right] \cdot Z \\
\text { s.t. } & A x=b, \quad \operatorname{Diag}\left(A X A^{\mathrm{T}}\right)=b \circ b, \\
& \sqrt{x^{\mathrm{T}} H x} \leqslant f^{\mathrm{T}} x, \quad\left(f f^{\mathrm{T}}-H\right) \cdot X \geqslant 0, \\
& Z=\left[\begin{array}{cc}
1 & x^{\mathrm{T}} \\
x & X
\end{array}\right] \succeq 0 .
\end{aligned}
$$

Consequently, the optimal value of problem (QP-SOC) can be computed in polynomial time using interior-point algorithms [15]. The optimal solution of problem (QP-SOC) is discussed in the next three subsections. 
By Definition $3.1, D_{\mathcal{L}}^{*}$ is the closed and convex cone generated by $\{X=$ $\left.\left[\begin{array}{l}1 \\ x\end{array}\right]\left[\begin{array}{l}1 \\ x\end{array}\right]^{\mathrm{T}} \in \mathcal{S}^{n+1}: x \in \mathcal{L}\right\}$. Denote

$$
\mathrm{Feas}(\mathrm{SP})=\{Z: Z \text { is feasible to }(\mathrm{SP})\} .
$$

Eichfelder and Povh [9] provided a theoretical proof for the existence of the decomposition form for any $Z \in \operatorname{Feas}(\mathrm{SP})$ :

Lemma 4.1 [9] For each feasible point $Z \in$ Feas(SP),

$$
Z=\sum_{i=1}^{p} \gamma_{i}\left[\begin{array}{c}
1 \\
x^{i}
\end{array}\right]\left[\begin{array}{c}
1 \\
x^{i}
\end{array}\right]^{\mathrm{T}}+\sum_{j=1}^{q} \beta_{j}\left[\begin{array}{c}
0 \\
d^{j}
\end{array}\right]\left[\begin{array}{c}
0 \\
d^{j}
\end{array}\right]^{\mathrm{T}},
$$

where $\gamma_{i}>0, \sum_{i=1}^{p} \gamma_{i}=1, \beta_{j}>0, x^{i} \in \mathcal{L}, d^{j} \in \mathcal{L}, A x^{i}=b$ and $A d^{j}=0$ for $i=$ $1, \cdots, p, j=1, \cdots, q$.

For simplicity, we call the vectors $x^{i}, i=1, \cdots, p$, and $d^{j}, j=1, \cdots, q$, as the decomposition vectors. However, Lemma 4.1 is a theoretical proof and only states the existence of a decomposition for $Z \in$ Feas(SP). It remains to be a problem of how to find the decomposition vectors. We will provide a polynomial-time constructive proof to obtain the decomposition of any $Z \in$ Feas(SP) under some proper conditions to generate an optimal solution or a recession direction of problem (QP-SOC).

4.2 Sufficient Conditions for Obtaining Decomposition Vectors in Polynomial Time

Notice that the optimal solution $x$ of problem (SP) is a feasible solution to problem (QP-SOC). If $\sqrt{x^{\mathrm{T}} H x}=f^{\mathrm{T}} x$, then $x$ is a boundary point of $\mathcal{L}$ and the direction along $x$ is an extreme ray of $\mathcal{L}$. If $\sqrt{x^{\mathrm{T}} H x}<f^{\mathrm{T}} x$, then $x$ is an interior point of $\mathcal{L}$, and $x$ can be represented as the convex combination of two boundary points as shown in the next lemma.

Lemma 4.2 Let $x$ be feasible to problem (QP-SOC) and $\tilde{V}=\left[\begin{array}{c}f^{\mathrm{T}} \\ x^{\mathrm{T}} H\end{array}\right]$. If there exists $d \in \mathbb{R}^{n}$ such that $\tilde{V} d=0$ and $d^{\mathrm{T}} H d=\left(f^{\mathrm{T}} x\right)^{2}-x^{\mathrm{T}} H x$, then $x=\frac{1}{2} v^{1}+\frac{1}{2} v^{2}$, where $v^{1}=x+d, v^{2}=x-d$ are boundary points of $\mathcal{L}$, i.e., $\sqrt{\left(v^{i}\right)^{\mathrm{T}} H v^{i}}=f^{\mathrm{T}} v^{i}, i=1,2$.

Proof We only need to prove $v^{1}$ and $v^{2}$ are boundary points of $\mathcal{L}$. Based on the selection of $d$,

$$
\begin{aligned}
\left(v^{i}\right)^{\mathrm{T}} H v^{i} & =(x \pm d)^{\mathrm{T}} H(x \pm d)=x^{\mathrm{T}} H x \pm 2 x^{\mathrm{T}} H d+d^{\mathrm{T}} H d \\
& =\left(f^{\mathrm{T}} x\right)^{2}=\left[f^{\mathrm{T}}(x \pm d)\right]^{2}=\left(f^{\mathrm{T}} v^{i}\right)^{2} .
\end{aligned}
$$

Since $H$ is positive definite and $f^{\mathrm{T}} x \geqslant 0, \sqrt{\left(v^{i}\right)^{\mathrm{T}} H v^{i}}=f^{\mathrm{T}} v^{i}, i=1,2$.

Remark 4.1 If $x$ is a boundary point of $\mathcal{L}$, i.e., $\sqrt{x^{\mathrm{T}} H x}=f^{\mathrm{T}} x$, then $d=0$ and $v^{1}=v^{2}=x$. Otherwise, since $\tilde{V}$ is a $2 \times n$-dimensional matrix, for $n>2$, 
the linear equation system $\tilde{V} d=0$ always has a nontrivial solution $\tilde{d}$, then $d=$ $\frac{\tilde{d}}{\sqrt{\tilde{d}^{\mathrm{T}} H \tilde{d}}} \sqrt{\left(f^{\mathrm{T}} x\right)^{2}-x^{\mathrm{T}} H x}$ satisfies $d^{\mathrm{T}} H d=\left(f^{\mathrm{T}} x\right)^{2}-x^{\mathrm{T}} H x$. Therefore, for any feasible solution $x$ of problem (QP-SOC), we can always find two boundary points to represent it.

Based on Lemma 4.2, we have the first sufficient condition as shown in the next theorem under which the decomposition vectors can be obtained in polynomial time.

Theorem 4.1 Let $Z=\left[\begin{array}{ll}1 & x^{\mathrm{T}} \\ x & x\end{array}\right]$ be a feasible solution of problem (SP). Assume the conditions in Lemma 4.2 are satisfied and $v^{1}=x+d, v^{2}=x-d$ be defined as in Lemma 4.2. If $X-x x^{\mathrm{T}} \succeq d d^{\mathrm{T}}$, then $Z$ can be decomposed in polynomial time as

$$
Z=\sum_{i=1}^{p} \gamma_{i}\left[\begin{array}{c}
1 \\
x^{i}
\end{array}\right]\left[\begin{array}{c}
1 \\
x^{i}
\end{array}\right]^{\mathrm{T}}+\sum_{j=1}^{q} \beta_{j}\left[\begin{array}{c}
0 \\
d^{j}
\end{array}\right]\left[\begin{array}{c}
0 \\
d^{j}
\end{array}\right]^{\mathrm{T}}
$$

where $\gamma_{i}>0, \sum_{i=1}^{p} \gamma_{i}=1, \beta_{j}>0, x^{i} \in \mathcal{L}, d^{j} \in \mathcal{L}, A x^{i}=b$ and $A d^{j}=0, i=$ $1, \cdots, p, j=1, \cdots, q$.

Proof For any feasible solution $Z=\left[\begin{array}{ll}1 & x^{\mathrm{T}} \\ x & x\end{array}\right]$ of problem (SP), $x$ is feasible to problem (QP-SOC). According to Lemma 4.2, there exist $v^{1}$ and $v^{2}$ such that $x=\frac{1}{2} v^{1}+\frac{1}{2} v^{2}$ and $\sqrt{\left(v^{i}\right)^{\mathrm{T}} H v^{i}}=f^{\mathrm{T}} v^{i}$, i.e., $\left(f f^{\mathrm{T}}-H\right)\left(v^{i}\left(v^{i}\right)^{\mathrm{T}}\right)=0$. Also, $\left(f f^{\mathrm{T}}-H\right) \cdot(X-$ $\left.\frac{1}{2} v^{1}\left(v^{1}\right)^{\mathrm{T}}-\frac{1}{2} v^{2}\left(v^{2}\right)^{\mathrm{T}}\right)=\left(f f^{\mathrm{T}}-H\right) \cdot X \geqslant 0$. Therefore,

$$
Z=\frac{1}{2}\left[\begin{array}{c}
1 \\
v^{1}
\end{array}\right]\left[\begin{array}{c}
1 \\
v^{1}
\end{array}\right]^{\mathrm{T}}+\frac{1}{2}\left[\begin{array}{c}
1 \\
v^{2}
\end{array}\right]\left[\begin{array}{c}
1 \\
v^{2}
\end{array}\right]^{\mathrm{T}}+\left[\begin{array}{cc}
0 & 0 \\
0 & X-\frac{1}{2} v^{1}\left(v^{1}\right)^{\mathrm{T}}-\frac{1}{2} v^{2}\left(v^{2}\right)^{\mathrm{T}}
\end{array}\right] .
$$

Denote $\tilde{X}=X-\frac{1}{2} v^{1}\left(v^{1}\right)^{\mathrm{T}}-\frac{1}{2} v^{2}\left(v^{2}\right)^{\mathrm{T}}$. If $X-x x^{\mathrm{T}} \succeq d d^{\mathrm{T}}$, then

$$
\begin{aligned}
\tilde{X} & =X-x x^{\mathrm{T}}-\left(\frac{1}{4} v^{1}\left(v^{1}\right)^{\mathrm{T}}+\frac{1}{4} v^{2}\left(v^{2}\right)^{\mathrm{T}}-\frac{1}{4} v^{1}\left(v^{2}\right)^{\mathrm{T}}-\frac{1}{4} v^{2}\left(v^{1}\right)^{\mathrm{T}}\right) \\
& =X-x x^{\mathrm{T}}-d d^{\mathrm{T}} \succeq 0 .
\end{aligned}
$$

According to Lemma 3.4, there exists a rank-one decomposition for $\tilde{X}$ such that $\tilde{X}=$ $\sum_{j=1}^{q} d^{j}\left(d^{j}\right)^{\mathrm{T}}$ and $\left(d^{j}\right)^{\mathrm{T}}\left(f f^{\mathrm{T}}-H\right) d^{j} \geqslant 0$ for $j=1, \cdots, q$, where $q=\operatorname{rank}(\tilde{X})$. We can choose $d^{j}$ such that $f^{\mathrm{T}} d^{j} \geqslant 0$, therefore, $\sqrt{\left(d^{j}\right)^{\mathrm{T}} H d^{j}} \leqslant f^{\mathrm{T}} d^{j}$, i.e., $d^{j} \in \mathcal{L}$. Thus,

$$
Z=\sum_{i=1}^{p} \gamma_{i}\left[\begin{array}{c}
1 \\
x^{i}
\end{array}\right]\left[\begin{array}{c}
1 \\
x^{i}
\end{array}\right]^{\mathrm{T}}+\sum_{j=1}^{q} \beta_{j}\left[\begin{array}{c}
0 \\
d^{j}
\end{array}\right]\left[\begin{array}{c}
0 \\
d^{j}
\end{array}\right]^{\mathrm{T}}
$$

where $\gamma_{i}>0, \sum_{i=1}^{p} \gamma_{i}=1, \beta_{j}>0, x^{i} \in \mathcal{L}$ and $d^{j} \in \mathcal{L}, i=1, \cdots, p, j=1, \cdots, q$. We now need to prove that $A x^{i}=b$ and $A d^{j}=0$. According to the constraints of 
problem (SP), for each row vector $\alpha^{k}$ of $A$, we have

$$
\sum_{i=1}^{p} \gamma_{i}\left(\alpha^{k}\right)^{\mathrm{T}} x^{i}=b_{k} \quad \text { and } \quad \sum_{i=1}^{p} \gamma_{i}\left(\left(\alpha^{k}\right)^{\mathrm{T}} x^{i}\right)^{2}+\sum_{j=1}^{q} \beta_{j}\left(\left(\alpha^{k}\right)^{\mathrm{T}} d^{j}\right)^{2}=b_{k}^{2}
$$

Consequently, according to Cauchy-Schwarz inequality,

$$
\left(\sum_{i=1}^{p} \gamma_{i}\right)\left(\sum_{i=1}^{p} \gamma_{i}\left(\left(\alpha^{k}\right)^{\mathrm{T}} x^{i}\right)^{2}+\sum_{j=1}^{q} \beta_{j}\left(\left(\alpha^{k}\right)^{\mathrm{T}} d^{j}\right)^{2}\right)=b_{k}^{2}=\left(\sum_{i=1}^{p} \gamma_{i}\left(\alpha^{k}\right)^{\mathrm{T}} x^{i}\right)^{2}
$$

holds if and only if

$$
\begin{gathered}
\left(\alpha^{k}\right)^{\mathrm{T}} d^{j}=0 \quad \text { and } \sqrt{\gamma_{i}}\left(\alpha^{k}\right)^{\mathrm{T}} x^{i}=\tau_{k} \sqrt{\gamma_{i}}, \\
i=1, \cdots, p, j=1, \cdots, q, k=1, \cdots, m,
\end{gathered}
$$

where $\tau_{k}=\tau_{k}\left(\sum_{i=1}^{p} \gamma_{i}\right)=\sum_{i=1}^{p} \gamma_{i}\left(\alpha^{k}\right)^{\mathrm{T}} x^{i}=b_{k}$, we have $A x^{i}=b$ and $A d^{j}=0$ for $i=1, \cdots, p, j=1, \cdots, q$.

Remark 4.2 If $x$ is a boundary point of $\mathcal{L}$, i.e., $\sqrt{x^{\mathrm{T}} H x}=f^{\mathrm{T}} x$, then $d=0$ and the condition of Theorem 4.1 is satisfied automatically. In this case, the decomposition vectors can be obtained in polynomial time without the assumptions in Lemma 4.2.

Theorem 4.1 depends on the existence of the vector $d$. The next sufficient condition depends on the solution of problem (SP) itself.

Theorem 4.2 For any feasible solution $Z=\left[\begin{array}{ll}1 & x^{\mathrm{T}} \\ x & X\end{array}\right]$ of problem (SP), there exists a rank-one decomposition for $X$ such that $X=\sum_{i=1}^{r} x^{i}\left(x^{i}\right)^{\mathrm{T}}$ and $x^{i} \in \mathcal{L}$ for $i=1, \cdots, r$, where $r=\operatorname{rank}(X)$. Moreover, if the optimal value of the following convex problem:

$$
\begin{aligned}
V_{\mathrm{OP}}:=\min & \|M \gamma-x\|_{2} \\
\text { s.t. } & \gamma \geqslant 0, \\
& \|\gamma\|_{2} \leqslant 1
\end{aligned}
$$

is 0 , where $M=\left[x^{1} ; \cdots ; x^{r}\right] \in \mathbb{R}^{n \times r}$, then $Z=\left[\begin{array}{cc}1 & x^{\mathrm{T}} \\ x & X\end{array}\right]$ can be decomposed as

$$
Z=\sum_{i=1, \gamma_{i}>0}^{r} \gamma_{i}^{2}\left[\begin{array}{c}
1 \\
\frac{x^{i}}{\gamma_{i}}
\end{array}\right]\left[\begin{array}{c}
1 \\
\frac{x^{i}}{\gamma_{i}}
\end{array}\right]^{\mathrm{T}}+\left(1-\|\gamma\|_{2}^{2}\right)\left[\begin{array}{l}
1 \\
0
\end{array}\right]\left[\begin{array}{l}
1 \\
0
\end{array}\right]^{\mathrm{T}}+\sum_{i=1, \gamma_{i}=0}^{r}\left[\begin{array}{c}
0 \\
x^{i}
\end{array}\right]\left[\begin{array}{c}
0 \\
x^{i}
\end{array}\right]^{\mathrm{T}} .
$$

Proof For any feasible solution $Z=\left[\begin{array}{ll}1 & x^{\mathrm{T}} \\ x & X\end{array}\right]$ of problem (SP), since $X \succeq 0$ and $\left(f f^{\mathrm{T}}-\right.$ $H) \cdot X \geqslant 0$, according to Lemma 3.4, there exists a rank-one decomposition for $X$ such that $X=\sum_{i=1}^{r} x^{i}\left(x^{i}\right)^{\mathrm{T}}$ and $\left(x^{i}\right)^{\mathrm{T}}\left(f f^{\mathrm{T}}-H\right) x^{i} \geqslant 0$ for $i=1, \cdots, r$, where $r=$ 
$\operatorname{rank}(X)$. We can choose $x^{i}$ such that $f^{\mathrm{T}} x^{i} \geqslant 0$, consequently, $\sqrt{\left(x^{i}\right)^{\mathrm{T}} H x^{i}} \leqslant f^{\mathrm{T}} x^{i}$, i.e., $x^{i} \in \mathcal{L}$. If $x=\sum_{i=1}^{r} \gamma_{i} x^{i}$, where $\gamma_{i} \geqslant 0, \sum_{i=1}^{r} \gamma_{i}^{2} \leqslant 1$, then $Z$ can be written as

$$
Z=\sum_{i=1, \gamma_{i}>0}^{r} \gamma_{i}^{2}\left[\begin{array}{c}
1 \\
\frac{x^{i}}{\gamma_{i}}
\end{array}\right]\left[\begin{array}{c}
1 \\
\frac{x^{i}}{\gamma_{i}}
\end{array}\right]^{\mathrm{T}}+\left(1-\|\gamma\|_{2}^{2}\right)\left[\begin{array}{l}
1 \\
0
\end{array}\right]\left[\begin{array}{l}
1 \\
0
\end{array}\right]^{\mathrm{T}}+\sum_{i=1, \gamma_{i}=0}^{r}\left[\begin{array}{c}
0 \\
x^{i}
\end{array}\right]\left[\begin{array}{c}
0 \\
x^{i}
\end{array}\right]^{\mathrm{T}}
$$

Similar to the proof of Theorem 4.1, we can show that $\frac{A x^{i}}{\gamma_{i}}=b$ for $i$ such that $\gamma_{i}>0$ and $A x^{i}=0$ for $i$ such that $\gamma_{i}=0$. Let $M=\left[x^{1} ; \cdots ; x^{r}\right]$, then $x$ can be represented as $x=\sum_{i=1}^{r} \gamma_{i} x^{i}$ such that $\gamma_{i} \geqslant 0$ and $\sum_{i=1}^{r} \gamma_{i}^{2} \leqslant 1$, if and only if $V_{\mathrm{OP}}=0$.

Note that the convex quadratic programming problem (OP) is polynomial-time solvable.

Remark 4.3 Under the conditions in Theorem 4.1, it can be seen through the proof that any feasible solution can be decomposed as in (4.3) with $p \leqslant 2$, while $p$ may be greater than 2 in Theorem 4.2. Therefore, the conditions in Theorem 4.1 do not dominate the conditions in Theorem 4.2. Moreover, the conditions in Theorem 4.2 are easier to be verified than those in Theorem 4.1. However, there are cases satisfying Theorem 4.1 but not satisfying Theorem 4.2. Examples are given in Sect. 5.

Denote the optimal solution of problem (SP) as $\bar{Z}$. If $V_{\mathrm{SP}}>-\infty$, for the decomposition of $\bar{Z}=\sum_{i=1}^{p} \gamma_{i}\left[\begin{array}{c}1 \\ \bar{x}^{i}\end{array}\right]\left[\begin{array}{c}1 \\ \bar{x}^{i}\end{array}\right]^{\mathrm{T}}+\sum_{j=1}^{q} \beta_{j}\left[\begin{array}{c}0 \\ \bar{d}^{j}\end{array}\right]\left[\begin{array}{c}0 \\ \bar{d}^{j}\end{array}\right]^{\mathrm{T}}$ as in (4.3), we have

$$
V_{\mathrm{SP}}=\sum_{i=1}^{p} \gamma_{i}\left(\left(\bar{x}^{i}\right)^{\mathrm{T}} Q \bar{x}^{i}+2 c^{\mathrm{T}} \bar{x}^{i}\right)+\sum_{j=1}^{q} \beta_{j}\left(\bar{d}^{j}\right)^{\mathrm{T}} Q \bar{d}^{j}
$$

We would like to find an optimal solution from this decomposition. Otherwise, a recession direction along which the objective value decreases to minus infinity is pursued. These two issues are addressed in the next two subsections, respectively.

\subsection{Optimal Solution When $V_{\mathrm{SP}}>-\infty$}

Theorem 4.3 If $V_{\mathrm{SP}}$ is finite with an attainable optimal solution $\bar{Z}=\sum_{i=1}^{p} \gamma_{i}\left[\begin{array}{c}1 \\ \bar{x}^{i}\end{array}\right] \times$ $\left[\begin{array}{c}1 \\ \bar{x}^{i}\end{array}\right]^{\mathrm{T}}+\sum_{j=1}^{q} \beta_{j}\left[\begin{array}{c}0 \\ \bar{d}^{j}\end{array}\right]\left[\begin{array}{c}0 \\ \bar{d}^{j}\end{array}\right]^{\mathrm{T}}$, then $\left(\bar{d}^{j}\right)^{\mathrm{T}} Q \bar{d}^{j}=0$ for $j=1, \cdots, q$, and $\bar{x}^{i}, i=$ $1, \cdots, p$, are optimal solutions of problem (QP-SOC).

Proof On one hand, if there exists a $k \in\{1, \cdots, q\}$ such that $\left(\bar{d}^{k}\right)^{\mathrm{T}} Q \bar{d}^{k}>0$, then $\sum_{i=1}^{p} \gamma_{i}\left[\begin{array}{c}1 \\ \bar{x}^{i}\end{array}\right]\left[\begin{array}{c}1 \\ \bar{x}^{i}\end{array}\right]^{\mathrm{T}}+\sum_{j=1, j \neq k}^{q} \beta_{j}\left[\begin{array}{c}0 \\ \bar{d}^{j}\end{array}\right]\left[\begin{array}{c}0 \\ \bar{d}^{j}\end{array}\right]^{\mathrm{T}} \in \mathrm{Feas}(\mathrm{SP})$ is a better solution than $\bar{Z}$ with the objective value reduced by $\beta_{k}\left(\bar{d}^{k}\right)^{\mathrm{T}} Q \bar{d}^{k}$. On the other hand, if there exists a $k \in\{1, \cdots, q\}$ such that $\left(\bar{d}^{k}\right)^{\mathrm{T}} Q \bar{d}^{k}<0$, then $\sum_{i=1}^{p} \gamma_{i}\left[\begin{array}{c}1 \\ \bar{x}^{i}\end{array}\right]\left[\begin{array}{c}1 \\ \bar{x}^{i}\end{array}\right]^{\mathrm{T}}+$ $\sum_{j=1, j \neq k}^{q} \beta_{j}\left[\begin{array}{c}0 \\ \bar{d}^{j}\end{array}\right]\left[\begin{array}{c}0 \\ \bar{d}^{j}\end{array}\right]^{\mathrm{T}}+\lambda\left[\begin{array}{c}0 \\ \bar{d}^{k}\end{array}\right]\left[\begin{array}{c}0 \\ \bar{d}^{k}\end{array}\right]^{\mathrm{T}} \in \mathrm{Feas}(\mathrm{SP})$ for any $\lambda>0$ with the objective 
value going to $-\infty$ as $\lambda \rightarrow+\infty$. This contradicts the finiteness of the objective optimal value. Therefore, $\left(\bar{d}^{j}\right)^{\mathrm{T}} Q \bar{d}^{j}=0$ for $j=1, \cdots, q$. Moreover,

$$
V_{\mathrm{SP}}=V_{\mathrm{QP}-\mathrm{SOC}} \leqslant \sum_{i=1}^{p} \gamma_{i}\left[\begin{array}{c}
1 \\
\bar{x}^{i}
\end{array}\right]^{\mathrm{T}}\left[\begin{array}{cc}
0 & c^{\mathrm{T}} \\
c & Q
\end{array}\right]\left[\begin{array}{c}
1 \\
\bar{x}^{i}
\end{array}\right]=\left[\begin{array}{cc}
0 & c^{\mathrm{T}} \\
c & Q
\end{array}\right] \cdot \bar{Z}=V_{\mathrm{SP}}
$$

Therefore, $\left[\begin{array}{c}1 \\ \bar{x}^{i}\end{array}\right]^{\mathrm{T}}\left[\begin{array}{cc}0 & c^{\mathrm{T}} \\ c & Q\end{array}\right]\left[\begin{array}{c}1 \\ \bar{x}^{i}\end{array}\right]=V_{\mathrm{QP}-S O C}$. Since $\bar{x}^{i}, i=1, \cdots, p$, are feasible to problem (QP-SOC), they are optimal solutions of problem (QP-SOC).

Notice that problem (SP) is a linear conic program and the cone involved is nonpolyhedral. Unlike the polyhedral cone, if $V_{\mathrm{SP}}$ is finite, the optimal solution may not be attainable $[13,16]$. However, the optimal value can be approximated by a sequence of linear conic programs with bounded feasible regions. Specifically, we can construct the following problem:

$$
\begin{aligned}
V_{\mathrm{SP}_{\mu}}:=\min & \bar{F}(Y)=\left[\begin{array}{ll}
0 & c^{\mathrm{T}} \\
c & Q
\end{array}\right] \cdot Z \\
\text { s.t. } & A x=b, \quad \operatorname{Diag}\left(A X A^{\mathrm{T}}\right)=b \circ b, \\
& \sqrt{x^{\mathrm{T}} H x} \leqslant f^{\mathrm{T}} x, \quad\left(f f^{\mathrm{T}}-H\right) \cdot X \geqslant 0, \\
& Z=\left[\begin{array}{cc}
1 & x^{\mathrm{T}} \\
x & X
\end{array}\right] \succeq 0, \quad\|Z\| \leqslant \mu,
\end{aligned}
$$

where $\mu>0$ is sufficiently large such that $\left|V_{\mathrm{SP}_{\mu}}-V_{\mathrm{SP}}\right|<\epsilon$ for a given $\epsilon>0$. If the optimal solution of problem $\left(\mathrm{SP}_{\mu}\right)$ can be decomposed according to Theorem 4.1 or 4.2 , then we can obtain an approximate solution of problem (QP-SOC). Examples are given in Sect. 5 .

\subsection{Recession Direction When $V_{\mathrm{SP}}=-\infty$}

When $V_{\mathrm{SP}}$ is infinite, we first consider the following problem:

$$
\begin{aligned}
V_{\mathrm{QP}-\mathrm{SOC}_{\infty}}:=\min & d^{\mathrm{T}} Q d \\
\text { s.t. } & A d=0, \\
& d \in \mathcal{L}, \quad\|d\|_{2} \leqslant K,
\end{aligned}
$$

where $K>0$ is a given constant to make the problem bounded. Then we have the next result.

Theorem 4.4 If $V_{\mathrm{SP}}=-\infty$ and $V_{\mathrm{QP}-\mathrm{SOC}_{\infty}}<0$, then $V_{\mathrm{QP}-\mathrm{SOC}} \rightarrow-\infty$ along the optimal solution of problem $\left(\mathrm{QP}-\mathrm{SOC}_{\infty}\right)$.

Proof If $V_{\mathrm{QP}-\mathrm{SOC}_{\infty}}<0$ with an optimal solution $d^{*}$, then $d^{*} \in 0^{+} \mathrm{Feas}(\mathrm{QP}-\mathrm{SOC})$. For any feasible solution $x \in$ Feas(QP-SOC), 


$$
\begin{aligned}
& \left(x+\lambda d^{*}\right)^{\mathrm{T}} Q\left(x+\lambda d^{*}\right)+2 c^{\mathrm{T}}\left(x+\lambda d^{*}\right) \\
& \quad=\left[\left(d^{*}\right)^{\mathrm{T}} Q d^{*}\right] \lambda^{2}+2\left(x^{\mathrm{T}} Q d^{*}+c^{\mathrm{T}} d^{*}\right) \lambda+x^{\mathrm{T}} Q x+2 c^{\mathrm{T}} x .
\end{aligned}
$$

Since $\left(d^{*}\right)^{\mathrm{T}} Q d^{*}<0$, the objective value at $x+\lambda d^{*}$ will go to $-\infty$ as $\lambda \rightarrow \infty$.

Theorem 4.4 provides a sufficient condition to find the direction along which the objective value of problem (QP-SOC) goes to $-\infty$. Notice that problem (QP-SOC $\infty$ ) is a quadratic programming problem and we only need to verify the sign of its optimal objective value. Similar to the detection of a copositive matrix [8] and the derivations of problem $\left(\mathrm{SP}_{\mu}\right)$, the sign of the optimal objective value of problem $\left(\mathrm{QP}-\mathrm{SOC}_{\infty}\right)$ is the same as the following semidefinite programming problem [23]:

$$
\begin{aligned}
V_{\mathrm{SP}_{\infty}}:=\min & \bar{F}(Y)=Q \cdot D \\
\text { s.t. } \quad & \operatorname{Diag}\left(A D A^{\mathrm{T}}\right)=0, \\
& \left(f f^{\mathrm{T}}-H\right) \cdot D \geqslant 0, \\
& D \succeq 0, \quad\|D\| \leqslant K^{2} .
\end{aligned}
$$

Therefore, when $V_{\mathrm{SP}}=-\infty$, we first solve problem $\left(\mathrm{SP}_{\infty}\right)$. If $V_{\mathrm{SP}_{\infty}}<0$ and the optimal solution $\left[\begin{array}{ll}1 & d^{\mathrm{T}} \\ d & D\end{array}\right]$ can be decomposed according to Theorem 4.1 or 4.2 , we can obtain a solution $d^{*}$ such that $\left(d^{*}\right)^{\mathrm{T}} Q d^{*}<0$ and $V_{\mathrm{QP}-\mathrm{SOC}} \rightarrow-\infty$ along $d^{*}$. Moreover, since $d$ is in the optimal solution of problem $\left(\mathrm{SP}_{\infty}\right)$, it is feasible to problem $\left(\mathrm{QP}-\mathrm{SOC}_{\infty}\right)$. If $d^{\mathrm{T}} Q d<0$, it is a direction along which $V_{\mathrm{QP}-\mathrm{SOC}}$ goes to $-\infty$.

\subsection{Polynomial-Time Algorithm for Problem (QP-SOC) with an Unbounded Feasible Region}

Based on the results obtained in Sects. 4.1 to 4.3, we can design a polynomial-time algorithm to solve problem (QP-SOC) with an unbounded feasible region under some proper conditions.

\section{Algorithm 2: Polynomial-time algorithm for problem (QP-SOC) with an un- bounded feasible region}

Step 1 For a given problem $(\mathrm{QP}-\mathrm{SOC})$, if Feas $(\mathrm{QP}-\mathrm{SOC})=\varnothing$, return that problem (QP-SOC) is infeasible. Otherwise, calculate the dual cone of $\mathcal{L}$, construct and solve the corresponding second-order programming problem (CPP). If the optimal value of problem (CPP) is negative, return that Feas(QP-SOC) is bounded and apply Algorithm 1 to solve the problem. Otherwise, construct problem (SP), record the optimal value $V_{\mathrm{SP}}$ and the optimal solution $\bar{Z}$ and go to Step 2 .

Step 2 If $V_{\mathrm{SP}}=-\infty$, go to Step 4. If $V_{\mathrm{SP}}>-\infty$ and the optimal solution $\bar{Z}$ of problem (SP) is unattainable, go to Step 3. Otherwise, the optimal solution $\bar{Z}$ of problem (SP) is attainable. If $\bar{Z}$ can be decomposed as $\bar{Z}=\sum_{i=1}^{p} \gamma_{i}\left[\begin{array}{c}1 \\ \bar{x}^{i}\end{array}\right]\left[\begin{array}{c}1 \\ \bar{x}^{i}\end{array}\right]^{\mathrm{T}}+$ $\sum_{j=1}^{q} \beta_{j}\left[\begin{array}{c}0 \\ \bar{d}^{j}\end{array}\right]\left[\begin{array}{c}0 \\ \bar{d}^{j}\end{array}\right]^{\mathrm{T}}$ according to Theorem 4.1 or 4.2 , return $\bar{x}^{i}, i=1, \cdots, p$, as the optimal solutions of problem (QP-SOC) with the optimal value being $V_{\mathrm{SP}}$. If the decomposition vectors cannot be obtained, return that the optimal objective value is $V_{\text {SP }}$ while the optimal solution is not found. 
Step 3 Construct and solve the linear conic program $\left(\mathrm{SP}_{\mu}\right)$ with $\mu>0$ being sufficiently large such that $\left|V_{\mathrm{SP}_{\mu}}-V_{\mathrm{SP}}\right|<\epsilon$ for a given $\epsilon>0$. If the optimal solution $\bar{Z}$ of problem $\left(\mathrm{SP}_{\mu}\right)$ can be decomposed as $\bar{Z}=\sum_{i=1}^{p} \gamma_{i}\left[\begin{array}{c}1 \\ \bar{x}^{i}\end{array}\right]\left[\begin{array}{c}1 \\ \bar{x}^{i}\end{array}\right]^{\mathrm{T}}+$ $\sum_{j=1}^{q} \beta_{j}\left[\frac{0}{\bar{d}^{j}}\right]\left[\begin{array}{c}0 \\ \bar{d}^{j}\end{array}\right]^{\mathrm{T}}$ according to Theorem 4.1 or 4.2 , return $\bar{x}^{i}, i=1, \cdots, p$, as the approximate optimal solutions of problem (QP-SOC) with the approximate optimal value being $V_{\mathrm{SP}_{\mu}}$. Otherwise, return that the optimal objective value is $V_{\mathrm{SP}}$ while the optimal solution is not found.

Step 4 Solve problem $\left(\mathrm{SP}_{\infty}\right)$ and record the optimal solution $\left[\begin{array}{ll}1 & d^{\mathrm{T}} \\ d & D\end{array}\right]$. If $d^{\mathrm{T}} Q d<$ 0 , return that $d$ is a direction along which $V_{\mathrm{QP}-S O C}$ goes to $-\infty$. If $\left[\begin{array}{ll}1 & d^{\mathrm{T}} \\ d & D\end{array}\right]$ can

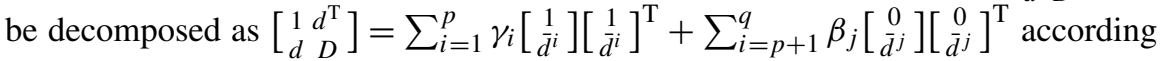
to Theorem 4.1 or 4.2 and $\left(\bar{d}^{k}\right)^{\mathrm{T}} Q \bar{d}^{k}<0$ for some $1 \leqslant k \leqslant q$, then return that $V_{\mathrm{QP}-\mathrm{SOC}} \rightarrow-\infty$ along $\bar{d}^{k}$. Otherwise, return that the optimal solution of problem is $-\infty$ while the recession direction is not found.

\section{Numerical Examples}

In this section, numerical examples are presented to illustrate the proposed approach. All examples were computed on a personal computer with $2.26 \mathrm{GHz}$ Intel Core 2 Duo CPU, 2 Gb of RAM, using Matlab R2012a-based SeDuMi solver [20] with a feasibility/optimality tolerance of $10^{-4}$.

Example 5.1 Consider the problem (QP-SOC) with

$$
\begin{aligned}
& Q=\left[\begin{array}{ccccc}
3.4031 & -0.5355 & -1.1798 & -0.5024 & -0.9224 \\
-0.5355 & -2.1305 & 0.9290 & -1.6814 & 1.0613 \\
-1.1798 & 0.9290 & 0.4346 & -0.7941 & -1.7420 \\
-0.5024 & -1.6814 & -0.7941 & 0.4129 & 0.2451 \\
-0.9224 & 1.0613 & -1.7420 & 0.2451 & -2.6656
\end{array}\right] \text {, } \\
& c=(-1.1691,0.1683,-3.4347,-0.8256,0.3751)^{\mathrm{T}} \text {, } \\
& A=\left[\begin{array}{ccccc}
-1.7311 & -0.6851 & -1.1302 & 1.1494 & 1.0574 \\
-2.2971 & 0.2635 & -0.2141 & -0.0409 & -0.5180
\end{array}\right] \text {, } \\
& b=(0.4889,0.9566)^{\mathrm{T}} \text {, } \\
& H=\left[\begin{array}{ccccc}
5.2532 & 4.3853 & 0.1132 & 1.6169 & 2.4867 \\
4.3853 & 10.0538 & -3.1039 & 0.5915 & 2.5201 \\
0.1132 & -3.1039 & 2.6282 & 1.4285 & 2.1709 \\
1.6169 & 0.5915 & 1.4285 & 2.2518 & 3.3347 \\
2.4867 & 2.5201 & 2.1709 & 3.3347 & 7.2601
\end{array}\right], \\
& f=(0.2372,0.2353,0.1533,0.9439,0.0412)^{\mathrm{T}} \text {. }
\end{aligned}
$$

The optimal value of problem (CPP) is $V_{\mathrm{CPP}}=-1<0$. By Corollary 2.2, the feasible region of this problem is bounded and we can use Algorithm 1 to solve 
problem (QP-SOC). A redundant constraint $\alpha^{\mathrm{T}} x=\left(A^{\mathrm{T}} k\right)^{\mathrm{T}} x=k^{\mathrm{T}} b$ can be constructed in Step 2, where $\alpha=(-32.2992,1.1939,-5.7635,2.7795,-3.1681)^{\mathrm{T}}$ and $k=(2.8422,11.9190)^{\mathrm{T}}$. By solving problem (LCoP1) in Step 3, the optimal solution

$$
\begin{aligned}
& {\left[\begin{array}{ll}
1 & y^{\mathrm{T}} \\
y & Y
\end{array}\right]} \\
& \quad=\left[\begin{array}{ccccc}
1.0000 & 58.6932 & 100.5050 & 452.2350 & -265.7160 \\
58.6932 & 3444.8952 & 5898.9601 & 26543.1930 & -15595.7223 \\
100.5050 & 5898.9601 & 10101.2550 & 45451.8787 & -26705.7866 \\
452.2350 & 26543.1930 & 45451.8787 & 204516.4952 & -120166.0753 \\
-265.7160 & -15595.7223 & -26705.7866 & -120166.0753 & 70604.9927
\end{array}\right]
\end{aligned}
$$

is obtained. Since $Y=y y^{\mathrm{T}}$, the optimal solution is rank-one and $y$ is an optimal solution of problem (QP-SOC2) according to Theorem 3.2. In Step 4 of the algorithm, an optimal solution $x=(48.8200,58.6932,100.5050,452.2350,-265.7160)^{\mathrm{T}}$ of problem (QP-SOC) is constructed with the optimal value $V_{\mathrm{QP}-\mathrm{SOC}}=-262683$.

Remark 5.1 Although the optimal value of this problem can also be obtained by solving problem (SP), the optimal solution of problem (SP)

$\left[\begin{array}{cccccc}1.0000 & 48.8199 & 58.6938 & 100.5034 & 452.2326 & -265.7147 \\ 48.8199 & 2383.3895 & 2865.4300 & 4906.5782 & 22077.9999 & -12972.1943 \\ 58.6938 & 2865.4300 & 3444.9632 & 5898.9336 & 26543.2752 & -15595.8205 \\ 100.5034 & 4906.5782 & 5898.9336 & 10100.9548 & 45450.9984 & -26705.2807 \\ 452.2326 & 22077.9999 & 26543.2752 & 45450.9984 & 204514.6530 & -120165.0436 \\ -265.7147 & -12972.1943 & -15595.8205 & -26705.2807 & -120165.0436 & 70604.4164\end{array}\right]$

is not a rank-one matrix and further decomposition is needed. Since the general method for rank-one decomposition is not fully explored, it is nontrivial job to obtain the optimal solution of the original problem. In contrast, the proposed algorithm in this paper can fully solve this problem in polynomial time.

Example 5.2 Consider the problem (QP-SOC) with

$$
\begin{aligned}
Q & =\left[\begin{array}{llll}
1 & 1 & 0 & 0 \\
1 & 1 & 0 & 0 \\
0 & 0 & 1 & 1 \\
0 & 0 & 1 & 1
\end{array}\right], \quad c=(-1,-1,0,0)^{\mathrm{T}}, \\
H & =I_{4}, \quad f=(0,0,0, \sqrt{2})^{\mathrm{T}}, \quad A=\left[\begin{array}{llll}
1 & -1 & 0 & 0
\end{array}\right], \quad b=0 .
\end{aligned}
$$

Since $\pm A^{\mathrm{T}} \notin \mathcal{L}^{*}$, the feasible region of this problem is unbounded and it is easy to verify that $(0,0,-1,1)^{\mathrm{T}}$ is a recession direction. By applying Algorithm 2 , the optimal solution of problem (SP) is unattainable but an approximate optimal objective value $V_{\mathrm{SP}_{\mu}}=-1.0000$ can be obtained by solving problem $\left(\mathrm{SP}_{\mu}\right)$ with $\mu=1500$ and the optimal solution is 


$$
\begin{aligned}
Z & =\left[\begin{array}{cc}
1 & x^{\mathrm{T}} \\
x & X
\end{array}\right] \\
& =\left[\begin{array}{ccccc}
1.0000 & 0.4999 & 0.4999 & -26.0852 & 26.0948 \\
0.4999 & 0.2499 & 0.2499 & -13.0404 & 13.0452 \\
0.4999 & 0.2499 & 0.2499 & -13.0404 & 13.0452 \\
-26.0852 & -13.0404 & -13.0404 & 698.0371 & -698.2936 \\
26.0948 & 13.0452 & 13.0452 & -698.2936 & 698.5503
\end{array}\right] .
\end{aligned}
$$

Note that $x$ is feasible to problem (QP-SOC) but $Z$ is not a rank-one matrix. Therefore, the optimal solution of problem (QP-SOC) cannot be obtained directly. However, $x$ is a boundary point of $\mathcal{L}$, and the solution $Z$ satisfies the conditions in Theorem 4.1. Then $Z$ can be decomposed as

$$
Z=\left[\begin{array}{cc}
1 & x^{\mathrm{T}} \\
x & X
\end{array}\right]=\left[\begin{array}{l}
1 \\
x
\end{array}\right]\left[\begin{array}{l}
1 \\
x
\end{array}\right]^{\mathrm{T}}+\left[\begin{array}{c}
0 \\
d_{1}
\end{array}\right]\left[\begin{array}{c}
0 \\
d_{1}
\end{array}\right]^{\mathrm{T}},
$$

where $d_{1}=(0,0,-4.1954,4.1969)^{\mathrm{T}}$. Since the optimal value is finite, according to Theorem $4.3, x=(0.4999,0.4999,-26.0852,26.0948)^{\mathrm{T}}$ is an approximate optimal solution of problem (QP-SOC).

Remark 5.2 Actually, the optimal solution of the problem is $x=\left(\frac{1}{2}, \frac{1}{2}, \alpha, \sqrt{\alpha^{2}+\frac{1}{2}}\right)^{\mathrm{T}}$ with $\alpha<0$ and $|\alpha|$ being arbitrarily large, which is unattainable and the optimal value is -1 [13]. However, when $\mu$ is sufficiently large in problem $\left(\mathrm{SP}_{\mu}\right)$, we can obtain an approximate solution. Since we can obtain the decomposition for the optimal solution of problem $\left(\mathrm{SP}_{\mu}\right)$, the decomposition vector $x$ is a good approximation to the optimal solution of problem (QP-SOC) in the sense that there is only a small difference between their objective values.

Remark 5.3 Note that we can also decompose $X$ according to Theorem 4.2 such that $X=\sum_{i=1}^{2} x^{i}\left(x^{i}\right)^{\mathrm{T}}$, where $x^{1}=(-0.0384,-0.0384,-2.1794,2.1801)^{\mathrm{T}}$ and $x^{2}=(0.4984,0.4984,-26.3304,26.3400)^{\mathrm{T}}$. By solving problem $(\mathrm{OP})$, the optimal value is 0.0087 and $Z$ does not satisfy the conditions of Theorem 4.2. Therefore, the conditions in Theorem 4.2 do not dominate the ones in Theorem 4.1.

Example 5.3 Consider the problem (QP-SOC) with

$$
\begin{aligned}
Q & =\left[\begin{array}{cccc}
0.8564 & 0.9360 & 0.3535 & 0.6963 \\
0.9360 & 1.3542 & 0.2730 & 0.0592 \\
0.3535 & 0.2730 & -2.9503 & 1.2095 \\
0.6963 & 0.0592 & 1.2095 & -0.7019
\end{array}\right], \\
c & =(0.8353,1.0369,-0.0669,-1.7341)^{\mathrm{T}}, \\
H & =\left[\begin{array}{cccc}
5.7625 & 0.1783 & 2.3100 & -0.9367 \\
0.1783 & 4.1657 & 1.1962 & -1.5644 \\
2.3100 & 1.1962 & 1.4195 & -1.2028 \\
-0.9367 & -1.5644 & -1.2028 & 1.6440
\end{array}\right],
\end{aligned}
$$




$$
\begin{aligned}
& f=(0.7621,0.4565,0.0185,0.8214)^{\mathrm{T}}, \\
& A=\left[\begin{array}{llll}
0.0000 & -0.3179 & 1.0950 & -1.8740
\end{array}\right], \quad b=0.4447 .
\end{aligned}
$$

Since $\pm A^{\mathrm{T}} \notin \mathcal{L}^{*}$, the feasible region of this problem is unbounded and $(-2.5486$, $-0.0200,8.2788,4.8408)^{\mathrm{T}}$ is a recession direction. The optimal value is also unbounded by solving problem $(\mathrm{SP})$. In this case, problem $\left(\mathrm{SP}_{\infty}\right)$ is constructed to find the direction along which the optimal value goes to minus infinity. By solving problem $\left(\mathrm{SP}_{\infty}\right)$ with $K=1000$, the optimal value is $V_{\mathrm{SP}_{\infty}}=-1519.5$. Hence, the optimal value goes to minus infinity along a direction based on Theorem 4.4. The optimal solution is

$$
\left[\begin{array}{ll}
1 & d^{\mathrm{T}} \\
d & D
\end{array}\right]=\left[\begin{array}{ccccc}
1.0000 & -0.0611 & 0.0046 & 0.2113 & 0.1227 \\
-0.0611 & 70.1757 & -12.1995 & -221.2273 & -127.1229 \\
0.0046 & -12.1995 & 2.1213 & 38.4584 & 22.0990 \\
0.2113 & -221.2273 & 38.4583 & 697.4191 & 400.7552 \\
0.1227 & -127.1229 & 22.0991 & 400.7551 & 230.2843
\end{array}\right],
$$

where $d$ is a feasible solution to problem $\left(\mathrm{QP}-\mathrm{SOC}_{\infty}\right)$ but not a boundary point of $\mathcal{L}$. However, $V_{\mathrm{OP}}=0, M=\left[d^{1} ; d^{2} ; d^{3}\right]$, where $d^{1}=(0.0182,-0.0196,0.0039$, $0.0051)^{\mathrm{T}}, d^{2}=(-0.1796,0.0169,0.5260,0.3041)^{\mathrm{T}}, d^{3}=(-8.3752,1.4562$, $26.4035,15.1721)^{\mathrm{T}}$ and $\gamma=(0.3315,0.0376,0.0072)^{\mathrm{T}}$. Therefore, according to Theorem 4.2, the optimal solution can be decomposed as

$$
\left[\begin{array}{cc}
1 & d^{\mathrm{T}} \\
d & D
\end{array}\right]=\sum_{i=1}^{3} \gamma_{i}^{2}\left[\begin{array}{c}
1 \\
\frac{d^{i}}{\gamma_{i}}
\end{array}\right]\left[\begin{array}{c}
1 \\
\frac{d^{i}}{\gamma_{i}}
\end{array}\right]^{\mathrm{T}}+\left(1-\|\gamma\|_{2}^{2}\right)\left[\begin{array}{l}
1 \\
0
\end{array}\right]\left[\begin{array}{l}
1 \\
0
\end{array}\right]^{\mathrm{T}} .
$$

Consequently, $d^{1}, d^{2}$ and $d^{3}$ are the directions along which the optimal solution goes to minus infinity.

The three examples show that the proposed approach can effectively solve problem (QP-SOC).

\section{Conclusion}

In this paper, we have studied the nonhomogeneous quadratic programming problem over a second-order cone with linear equality constraints. We dealt with the problem depending on the boundedness of the feasible region. When the feasible region is bounded, the problem is reformulated as a linear conic program on the cone of nonnegative quadratic functions over a convex quadratic constraint, which can then be solved in polynomial time to obtain an optimal solution of the original problem. When the feasible region is unbounded, the problem is reformulated as an SDP problem and the optimal objective value of the original problem can be obtained in polynomial time. Two sufficient conditions are derived, under which, if the optimal objective value is finite, an optimal solution of the original problem can be obtained by decomposing the optimal solution of the SDP problem into the original feasible 
region in polynomial time. Otherwise, a recession direction along which the optimal objective value goes to minus infinity can be found in polynomial time. Numerical examples are included to illustrate the effectiveness of the proposed algorithms. The results obtained in this paper may motivate the study of quadratic optimization problems over a more general cone in the future.

\section{References}

[1] Alizadeh, F., Goldfarb, D.: Second-order cone programming. Math. Program. 95, 3-51 (2003)

[2] Andersen, E.D., Roos, C., Terlaky, T.: Notes on duality in second order and $p$-order cone optimization. Optimization 51, 627-643 (2002)

[3] Ben-Tal, A., Nemirovski, A.: Lectures on Modern Convex Optimization, Analysis, Algorithms, and Engineering Applications. MPS/SIAM Series on Optimization. SIAM, Philadelphia (2001)

[4] Bishop, E., Phelps, R.R.: The support functionals of a convex set. Proc. Symp. Pure Math. 7, 27-35 (1962)

[5] Burer, S.: On the copositive representation of binary and continuous nonconvex quadratic programs. Math. Program. 120, 479-495 (2009)

[6] Burer, S., Anstreicher, K.M.: Second-order-cone constraints for extended trust-region subproblems. SIAM J. Optim. 23, 432-451 (2013)

[7] Cui, X.T., Zheng, X.J., Zhu, S.S., Sun, X.L.: Convex relaxations and MIQCQP reformulations for a class of cardinality-constrained portfolio selection problems. J. Glob. Optim. 56, 1409-1423 (2013)

[8] Deng, Z., Fang, S.-C., Jin, Q., Xing, W.: Detecting copositivity of a symmetric matrix by an adaptive ellipsoid-based approximation scheme. Eur. J. Oper. Res. 229, 21-28 (2013)

[9] Eichfelder, G., Povh, J.: On the set-semidefinite representation of nonconvex quadratic programs with cone constraints. Croat. Oper. Res. Rev. 1, 26-39 (2010)

[10] Eichfelder, G., Povh, J.: On the set-semidefinite representation of nonconvex quadratic programs over arbitrary feasible sets. Optim. Lett. 7, 1373-1386 (2013)

[11] Fang, S.-C., Xing, W.: Linear Conic Programming. Science Press, Beijing (2013) (in Chinese)

[12] Guo, X., Deng, Z., Fang, S.-C., Xing, W.: Quadratic optimization over one first-order cone. J. Ind. Manag. Optim. 10, 945-963 (2014). doi:10.3934/jimo.2014.10.945

[13] Jin, Q., Fang, S.-C., Xing, W.: On the global optimality of generalized trust region subproblems. Optimization 59, 1139-1151 (2010)

[14] Jin, Q., Tian, Y., Deng, Z., Fang, S.-C., Xing, W.: Exact computable representation of some secondorder cone constrained quadratic programming problems. J. Oper. Res. Soc. China 1, 107-134 (2013)

[15] Nesterov, Y., Nemirovsky, A.: Interior-Point Polynomial Methods in Convex Programming. SIAM, Philadelphia (1994)

[16] Parida, J., Roy, K.L.: An existence theorem for quadratic programs over cone domains. J. Appl. Math. Mech. 62, 207-208 (1982)

[17] Rockafellar, R.T.: Convex Analysis, 2nd edn. Princeton University Press, Princeton (1972)

[18] Saaty, T.L.: Fundamentals of the analytic network process multiple networks with benefits, costs, opportunities and risks. J. Syst. Sci. Syst. Eng. 13, 348-379 (2004)

[19] Strang, G.: Introduction to Applied Mathematics. Wellesley-Cambridge Press, Wellesley (1986)

[20] Sturm, J.F.: Using SeDuMi 1.02, a MATLAB toolbox for optimization over symmetric cones. Optim. Methods Softw. 11, 625-653 (1999)

[21] Sturm, J.F., Zhang, S.: On cones of nonnegative quadratic functions. Math. Oper. Res. 28, 246-267 (2003)

[22] Tian, Y., Fang, S.-C., Deng, Z., Xing, W.: Computable representation of the cone of nonnegative quadratic forms over a general second-order cone and its application to completely positive programming. J. Ind. Manag. Optim. 9, 701-709 (2013)

[23] Ye, Y., Zhang, S.: New results on quadratic minimization. SIAM J. Optim. 14, 245-267 (2003) 